Check for updates

Cite this: RSC Adv., 2017, 7, 18917

Received 5th February 2017

Accepted 21st March 2017

DOI: $10.1039 / \mathrm{c} 7 \mathrm{ra0} 01464 \mathrm{c}$

rsc.li/rsc-advances

\title{
Long-term antibacterial protected cotton fabric coating by controlled release of chlorhexidine gluconate from halloysite nanotubes $\uparrow$
}

\author{
Yu Wu, Yongtao Yang, Haoyang Liu, Xihui Yao, Fan Leng, (D) Yun Chen \\ and Weiqun Tian (D)*
}

\begin{abstract}
In this work, an environmentally friendly antibacterial composite was prepared by loading natural halloysite nanotubes (HNTs) with chlorhexidine gluconate (CG). Fourier transform-infrared spectroscopy and X-ray diffraction demonstrated that CG could be successfully loaded on HNTs without changing the crystalline structure of HNTs. In vitro drug release assay showed that CG could be released from HNTs in a sustainable manner and could last as long as about ten days. Inhibition zone test suggested that the new composite displayed significant bacterial killing activity. In addition, the composite was deposited on the surface of cotton fabric by dip-coat technique. Our results showed that the HNTs/CG composite could be distributed on the cotton fabric and possessed better thermal stability and hydrophilicity properties than raw cotton fabric. HNTs/CG-coated cotton fabric exhibited over $98 \%$ bacterial reduction of Staphylococcus aureus, Escherichia coli, and Pseudomonas aeruginosa, and still showed over 90\% antibacterial activity even after being washed 20 times. MTT test and animal skin irritation tests indicate that HNTs/CG-coated cotton was nontoxic to mouse fibroblast cells (L929) and gave no irritation to the rabbit's skin. Thus, HNTs/CG composite is a safe and promising antibacterial agent and has great potential application in the textile field.
\end{abstract}

\section{Introduction}

Owing to their outstanding properties of air permeability, comfort and softness, cotton textiles are widely used as medical and household products in our daily life. However, under appropriate temperature and humidity, the natural cotton fiber is beneficial to the rapid growth of microorganisms. ${ }^{1}$ This bacterial pollution leads to the loss of mechanical strength of the textiles, and is also a threat to human health. Therefore, there are high demands for environmentally friendly, cost effective and biocompatible antibacterial agents to impart antibacterial and antifouling properties to cotton fabrics.

In recent years, lots of studies have been performed to improve the antibacterial property of cotton fabrics by combining with different kinds of metal nanoparticles, such as Ag nanoparticles, ${ }^{2-5} \mathrm{TiO}_{2},{ }^{6,7}$ and $\mathrm{ZnO}^{8,9}$ Although these nanoparticles have good antibacterial effect, safety and environmental issues caused by the release of heavy metal ions are concerned and limit their widespread use. ${ }^{10}$ Compared with those metal nanoparticles, natural nanoparticles are desirable

Department of Biomedical Engineering, School of Basic Medical Sciences, Wuhan University, Wuhan 430071, P. R. China. E-mail: tian_weiqun@whu.edu.cn; Fax: +86 2768759991; Tel: +862768759509

$\dagger$ Electronic supplementary information (ESI) available. See DOI: 10.1039/c7ra01464c materials to use with the aim of developing new antibacterial textile products without environmental hazards or biological toxicity for the textile industry.

Halloysite nanotubes (HNTs) are a kind of hydrated clay nanotubes, which have deserved considerable interest owing to their low cost, environmentally friendly nature and readily availability. ${ }^{11}$ Owing to their peculiar hollow tubular shape, they exhibit high aspect ratio, good adsorbing ability and cation exchange capacity. The biocompatibility of halloysite is an issue of concern for the application of HNTs. ${ }^{12,13}$ The low toxicity of HNTs has been demonstrated by several studies. Ahmed et al. ${ }^{14}$ studied the cytotoxicity and cytogenetic toxicity of HNTs as oral drug delivery system, indicating HNTs did not cause toxicity to HCT116, HepG2 cells and human peripheral blood lymphocytes. Vergaro et al. ${ }^{15}$ investigated the interaction between HNTs and different cells for toxicity tests, and observed the uptake process of nanotubes into cells, representing a high level of biocompatibility of HNTs. Because of the high biocompatibility and low toxicity, HNTs can be used in food, biomedical and industrial fields, such as food packaging, ${ }^{16}$ tissue engineering scaffold, ${ }^{17}$ cancer cell isolation, ${ }^{18}$ bone implants, ${ }^{19}$ and cosmetics. ${ }^{20}$

It is identified that an ideal antimicrobial finishing agent should have broad-spectrum antimicrobial activity, low toxicity, no negative effect on the original properties of fabric, and exhibit excellent antimicrobial durability against washing. ${ }^{21,22}$ As a natural clay mineral, HNTs exhibit no antibacterial effect unless 
antibacterial drugs are intercalated. ${ }^{23}$ Chlorhexidine gluconate (CG) is an efficient antibacterial agent, which has broadspectrum antibacterial activity and low toxicity. ${ }^{24}$ As the positive charge of the chlorhexidine molecule will interact with the negatively charged phosphate groups on microbial cell walls, the electrostatic interaction can lead to the disruption of cellular membrane permeability. ${ }^{25}$ Some studies have demonstrated that CG-impregnated cloth has an obvious long-lasting antiseptic effect, as CG can persist on skin and is not inactivated by blood or serum proteins. ${ }^{26}$ Thus, intercalating the organic CG into inorganic HNTs may be an efficient way to obtain a novel composite antibacterial material. Moreover, the HNTs/CG composite as an antibacterial material coating on cotton fabric has not, to our knowledge, been reported previously.

In this paper, HNTs/CG composite material was prepared by loading CG in HNTs by vacuum suction method and then was coated on cotton fabric. The objectives of this research were to obtain an innovative cotton fabric which possesses long-term antibacterial properties. Fourier transform-infrared spectroscopy (FTIR) and X-ray diffraction (XRD) were used to characterize the new composite. In vitro drug release and inhibition zone test were performed to detect the controlled release performance and antibacterial activity of the composite material. To characterize the morphology of the cotton fabric, scanning electron microscopy (SEM) was employed. Finally, the effects of the HNTs/CG-coated cotton content on the thermal stability, hydrophilicity, antibacterial activity and biocompatibility are discussed in detail.

\section{Experimental section}

\section{Materials}

Halloysite was obtained from Jinyangguang Ceramic Co., Ltd (Zhengzhou, P. R. China). Chlorhexidine gluconate was purchased from Jingchuchen Pharmaceutical Chemical Co., Ltd (Wuhan, P. R. China). The structural formula of chlorhexidine gluconate is shown in Fig. S1.† The test bacteria, Escherichia coli, Pseudomonas aeruginosa and Staphylococcus aureus, used for this study were purchased from the China Center for Type Culture Collection. The Gram-positive $S$. aureus strain selected in the experiment was CCTCC AB 91093, the Gram-negative $E$. coli strain selected in the experiment was CCTCC AB 93154 and the $P$. aeruginosa strain selected in the experiment was CCTCC $\mathrm{AB}$ 93066. All of the chemical reagents used were analytical grade.

\section{Drug loading procedure}

The purchased HNTs were pretreated according to the protocols described in the literature. ${ }^{27}$ An ethyl alcohol (absolute) suspension of $0.5 \mathrm{~g}$ HNT powder was mixed with a saturated solution of CG drug and then evacuated for $30 \mathrm{~min}$ using a vacuum pump. Air bubbles were removed from the pores of HNTs to ensure the antibacterial agents could enter the lumens under negative pressure. After $30 \mathrm{~min}$, the vacuum had vanished and air was allowed into the container when back to atmospheric pressure. The process was repeated twice for the most efficient loading. Finally, the drug loaded samples were separated from the solution by centrifugation (9000 rpm) and were washed three times with deionized water to remove unloaded drug. Finally, HNTs/CG composite was dried in an oven at $60{ }^{\circ} \mathrm{C}$ and milled to a fine powder.

\section{Preparation of HNTs/CG-coated cotton fabric}

The HNTs/CG composite was coated on fabric by using the dipcoat method. HNTs/CG powder was added to deionized water and stirred vigorously. Then, the clean cotton fabric was impregnated in HNTs/CG suspension at room temperature, and then the excess liquid was squeezed out of the fabric. After being dried in an oven at $60{ }^{\circ} \mathrm{C}$, the HNTs/CG-coated cotton fabric was obtained.

\section{Structural characterization}

Transmission electron microscopy (TEM) images of HNTs were obtained from a Tecnai 12 Transmission Electron Microscope (FEI, Eindhoven, The Netherlands) equipped with a Megaview III CCD camera and analysis camera control software (Olympus) at an operating voltage of $120 \mathrm{kV}$.

Fourier transform-infrared (FTIR) spectra of samples were recorded on an FTIR spectrometer (TNZI-5700, Nicolet, USA) with the wavenumber range of 4000 to $400 \mathrm{~cm}^{-1}$ using $\mathrm{KBr}$ pellets. The crystal structure of HNT powder before and after CG loading was analyzed by an X-ray diffractometer (D8 ADVANCE, Germany) with $\mathrm{Cu} \mathrm{K} \alpha$ radiation $(\gamma=0.15406 \mathrm{~nm})$. XRD data were recorded from $4^{\circ}$ to $80^{\circ}(2 \theta)$ at a scanning speed of $1^{\circ} \mathrm{min}^{-1}$.

\section{In vitro drug release}

Drug release from HNTs was investigated in deionized water at room temperature. Three milliliters of pure CG drug $(10 \mathrm{mg}$ $\mathrm{mL}^{-1}$ ) was loaded into a dialysis bag and then dipped in $30 \mathrm{~mL}$ water. The halloysite nanotubes were dispersed in $10 \mathrm{~mL}$ water and constantly oscillated at room temperature. Then, the suspension was centrifuged to separate the sample for analysis. The concentration of CG was determined by UV spectrophotometry (PerkinElmer Lambda 25, USA). To estimate the complete release profile, halloysite samples were sonicated for $1 \mathrm{~h}$ at the end of each release study. ${ }^{28}$ All experiments were performed in triplicate.

\section{Inhibition zone test of HNTs/CG composite}

The antibacterial activity of HNTs/CG powder against $S$. aureus, $E$. coli and $P$. aeruginosa was tested through inhibition zone test. One hundred microliters of bacterial suspension with a concentration of $10^{5} \mathrm{CFU} \mathrm{mL}^{-1}$ was spread on Luria-Bertani (LB) agar plates, and then three holes were punched in each plate. HNTs/CG powder was put into the holes and then the plates were incubated at $37^{\circ} \mathrm{C}$. The diameter of inhibition zone was measured after $24 \mathrm{~h}$.

\section{Characterization of HNTs/CG-coated cotton fabric}

The morphology of the raw cotton fabric and the HNTs/CGcoated cotton fabric was characterized by scanning electron 
microscopy (SEM; VEGA3, TESCAN, Czech Republic) at a $20 \mathrm{kV}$ accelerating voltage. Thermogravimetric analysis (TGA) was carried out on a Diamond TG/DTA instrument (PerkinElmer, USA) under nitrogen atmosphere with a flow capacity of $20 \mathrm{~mL}$ $\min ^{-1}$. The scan was performed over the temperature range from 40 to $700{ }^{\circ} \mathrm{C}$ at a heating rate of $10^{\circ} \mathrm{C} \mathrm{min}^{-1}$.

\section{Evaluation of hydrophilicity of HNTs/CG-coated cotton}

The hydrophilicity of raw cotton textiles and HNTs/CG-coated cotton was evaluated on the basis of textile-test method for capillary effect (FZ/T 01071-2008). Three specimens were cut with a size of $250 \mathrm{~mm}$ length and $30 \mathrm{~mm}$ width. Thereafter, one side of each specimen was fixed on a bracket and the other side of the specimen was vertically suspended $15 \mathrm{~mm} \pm 2 \mathrm{~mm}$ below the zero point of a ruler. Deionized water with red color was poured into the container below the zero point of ruler, and the bottom of the specimen was immersed in the water. The water wicking height of specimen was recorded at different times. Each test was repeated in triplicate to calculate the mean value.

\section{Antibacterial tests of HNTs/CG-coated cotton fabric}

The antimicrobial activity of the HNTs/CG-coated cotton fabric was tested against the Gram-positive $S$. aureus and the Gramnegative $E$. coli and $P$. aeruginosa by the viable cell counting technique. In order to quantitatively analyze the antibacterial activity of the HNTs/CG-coated cotton fabric, antibacterial rate was used to represent the antibacterial effect. Bacteria were incubated in Luria-Bertani (LB) liquid nutrient medium for $18 \mathrm{~h}$ at $37^{\circ} \mathrm{C}$, and the number of cells was diluted to $10^{5}$ to $10^{6} \mathrm{CFU}$ $\mathrm{mL}^{-1}$ by standard serial dilution method. The coated cotton and the raw cotton were cut into pieces with an area of $1 \mathrm{~cm}^{2}$, and then dipped into the bacterial dilution mentioned above $(5 \mathrm{~mL})$. After incubation for $24 \mathrm{~h}, 100 \mu \mathrm{L}$ bacterial suspension was taken out and diluted to appropriate concentration. The dilution suspension was transferred onto agar plates, and then incubated at $37^{\circ} \mathrm{C}$ for $24 \mathrm{~h}$. The numbers of viable colonies were counted. The antibacterial rate was calculated by the following equation:

$$
\text { Antibacterial rate }=(1-B / A) \times 100 \%
$$

where $A$ is the number of bacterial colonies on the plates treated with the raw cotton fabric suspension and $B$ is the number of bacterial colonies on the plates treated with the suspension from the cotton fabric finished with HNTs/CG.

The bacterial cell viability was detected based on fluorescein isothiocyanate (FITC) and propidium iodide (PI) double stain. FITC remains on the outermost intact cell walls and produces a green color, whereas PI cannot penetrate the membrane of living bacterials, and can only bind to the DNA of damaged cells, giving a red color. Five microliters of FITC and $10 \mu \mathrm{L}$ PI stain were mixed to co-culture with a bacterial sample for $10 \mathrm{~min}$ at room temperature. Then, the sample was rinsed to remove the uncombined stain and observed under a fluorescence microscope (Olympus IX 73 DP80).

\section{Durability test}

The antibacterial rate was calculated after 20 washing cycles to evaluate the durability of HNTs/CG-coated cotton fabric against repeated laundering. The textile sample was washed with $2 \mathrm{~g}$ $\mathrm{L}^{-1}$ non-ionic detergent at $40{ }^{\circ} \mathrm{C}$ for $10 \mathrm{~min}$, then rinsed with clean water once and dehydrated, which was recorded as a washing cycle. The above steps were repeated to 20 washing times. To avoid the interference of washing agent, the textile sample was thoroughly washed with a large amount of water and dried before the antibacterial test.

\section{Skin irritation test}

All experiments were performed in compliance with the relevant laws and institutional guidelines such as the Ministry of Science and Technology of the People's Republic of China "Guidelines on the Treatment of Animals" (09/03/2006). We took great efforts to reduce the number of animals used in these studies and also to reduce animals' suffering pain and discomfort. All procedures concerning animals were approved by and conformed to the guidelines of the Animal Care \& Welfare Committee of Wuhan University School of Medicine. The skin irritation test was carried out according to a technical standard for disinfection (Ministry of Health of P. R. China, 2002). Three New Zealand rabbits were chosen as test animals and their hair was removed before testing. HNTs/CG suspension $(0.5 \mathrm{~mL})$ was added to the skin on one side of each rabbit, and covered with 4 layers of gauze. The skin on the other side of the rabbit served as a control. The irritation response (erythema and edema) of the skin was observed after $1 \mathrm{~h}, 24 \mathrm{~h}$, $48 \mathrm{~h}$ and $72 \mathrm{~h}$. The score of skin irritation for each tested rabbit was evaluated.

\section{Cytotoxicity test}

The cytotoxicity of the HNTs/CG-coated cotton was detected by MTT (3-(4,5-dimethylthiazol-2-yl)-2,5-diphenyltetrazolium bromide) assay with L929 mouse fibroblast cells. Cells were cultured in MEM-Alpha medium supplemented with $10 \%$ fetal bovine serum (FBS), and incubated at $37^{\circ} \mathrm{C}$ in a wet atmosphere containing $5 \% \mathrm{CO}_{2}$. The tested cotton fabric was cut into $2.5 \mathrm{~cm}$ $\times 2.5 \mathrm{~cm}$ pieces and soaked in $2 \mathrm{~mL}$ culture medium with no FBS for 3 days. The extracts were diluted to a concentration of $25 \%$ with culture medium. The L929 cells were seeded in 96well plates at a density of 2000 cells per well and incubated for $24 \mathrm{~h}$. Then, the medium was removed and replaced by prepared extracted dilutions. After incubation for 24,48 and $72 \mathrm{~h}$, the cells were treated with $20 \mu \mathrm{L}$ per well MTT solution $\left(5 \mathrm{mg} \mathrm{mL}^{-1}\right.$ in phosphate buffered saline, PBS) and incubated for another $4 \mathrm{~h}$ at $37^{\circ} \mathrm{C}$. Then, $200 \mu \mathrm{L}$ per well dimethyl sulfoxide (DMSO) was added to dissolve the formazan crystals. The plates were shaken for $15 \mathrm{~min}$, and the optical density was detected on a multiwell microplate reader (Tecan GENios, Tecan Austria $\mathrm{GmbH}$, Salzburg, Austria) at $490 \mathrm{~nm}$. Cell viability was calculated by the following equation:

$$
\text { Cell viability }=\mathrm{OD}_{\text {sample }} / \mathrm{OD}_{\text {control }} \times 100 \%
$$


where $\mathrm{OD}_{\text {sample }}$ is the absorbance of the test sample and $\mathrm{OD}_{\text {control }}$ is the absorbance for untreated control cells.

\section{Results and discussion}

\section{Morphology of HNTs observed by TEM}

The shape and size of the HNTs have an important effect on the loading efficiency and controlled release of the drug. So the morphology of halloysite nanotubes was observed through transmission electron microscopy (TEM). As shown in Fig. 1, the halloysite nanotubes dispersed evenly. The size of the nanotubes varied in the range $0.2-1.5 \mu \mathrm{m}$ length, $10-20 \mathrm{~nm}$ inner diameter, and 20-50 nm external diameter. Halloysite was revealed to be a cylindrical tube with an open-ended lumen and a transparent central area along the nanotube. The morphology of the halloysite nanotubes was identical to that reported in other literature. ${ }^{29}$ Because of the unique structure, halloysite has been deemed a promising nanoscale container for the encapsulation and delivery of biologically active molecules. ${ }^{30}$ Various drugs in concentrated solution or melt could be loaded in halloysite nanotubes. ${ }^{31}$

\section{FTIR and XRD analysis}

The successful loading of CG onto/into the HNTs was confirmed by FTIR spectroscopy (Fig. 2a). For neat HNTs, the bands at 3695 $\mathrm{cm}^{-1}$ and $3621 \mathrm{~cm}^{-1}$ were assigned to the stretching vibration of the inner-surface $\mathrm{Al}_{2} \mathrm{OH}$ groups, each $-\mathrm{OH}$ being linked to two $\mathrm{Al}$ atoms. The peak at $1633 \mathrm{~cm}^{-1}$ was attributed to bending vibration of interlayer water. The bands observed at $1091 \mathrm{~cm}^{-1}$ and $1032 \mathrm{~cm}^{-1}$ correspond to the in-plane stretching vibration of the $\mathrm{Si}-\mathrm{O}-\mathrm{Si}$. The band at $911 \mathrm{~cm}^{-1}$ was ascribed to the $\mathrm{O}-\mathrm{H}$ bending vibration of inner Al-OH groups. ${ }^{32}$ The bands for bending vibration of $\mathrm{Al}-\mathrm{O}-\mathrm{Si}$ and $\mathrm{Si}-\mathrm{O}-\mathrm{Si}$ were at $553 \mathrm{~cm}^{-1}$ and $471 \mathrm{~cm}^{-1}$, respectively. ${ }^{33}$ Compared with neat HNTs, the HNTs/ CG composite had both the characteristic bands of neat HNTs, and the absorption peaks of N-H of CG at $1533 \mathrm{~cm}^{-1}$ and 1492 $\mathrm{cm}^{-1}$. The results suggested that the loading of CG molecules onto/into HNTs is a physical adsorption process and without any chemical deterioration of functional groups. The possible reason is that CG is a kind of cationic drug, which can be adsorbed on the negatively charged outer surface of HNTs. In this manner, the electrostatic interactions between CG and
HNTs would contribute to adsorbing CG on the outer surface of HNTs, and more extensively entrapping it inside the hollow lumen under vacuum. ${ }^{34-36}$

The crystals structures of HNTs and HNTs/CG composite were characterized by XRD (Fig. 2b). The results showed that HNTs exhibit a diffraction peak at $11.91^{\circ}$ in $2 \theta$, which is the characteristic peak of halloysite ( $7 \AA$ A-form). The observed diffraction peaks of the HNTs/CG composite were consistent with neat HNTs, and the interlayer distance of HNTs and HNTs/ CG composite remained unchanged, indicating that the loading process of CG did not change the crystalline structure of HNTs.

\section{In vitro drug release}

The release curves of both CG from HNTs/CG composite and pure CG drug from a dialysis bag are presented in Fig. 3. Within the first hour, $75 \%$ of drug was released from the dialysis bag; the release curve of pure drug exhibited an initial burst release. After 4 hours, the pure drug CG was almost completely released into the water. In contrast, the HNTs/CG composite showed a relatively sustained drug release profile; only $25 \%$ of CG was released from nanotubes within the first hour. The release of this drug could last nearly ten days. The sustained drug release may be based on the small size of HNTs which confined the drug diffusion. As the drug molecules can be both intercalated in the lumen and adsorbed on the external surface of the clay nanotubes, the release kinetics represent the sum of drugs that were contained in the nanotubes and those released from the outer surface of the particles with a much slower release rate. ${ }^{37}$ The intensity of the antibacterial effect would depend on drug concentration released from carriers. The sustained release could prolong the effective time in contact with bacteria and lead to the amount of drug released reaching a level that could kill bacteria at the site of action. Raso et al. ${ }^{38}$ reported a controlled release system of chlorhexidine/porous silica compounds which exhibited excellent inhibitory concentration to kill bacteria due to the sustainable release of drug into the medium, instead of by direct contact with antibacterial compounds.

\section{Inhibition zone test of HNTs/CG composite}

The bacterial inhibition efficacy of HNTs/CG composite was tested by qualitative analysis with the agar diffusion plate
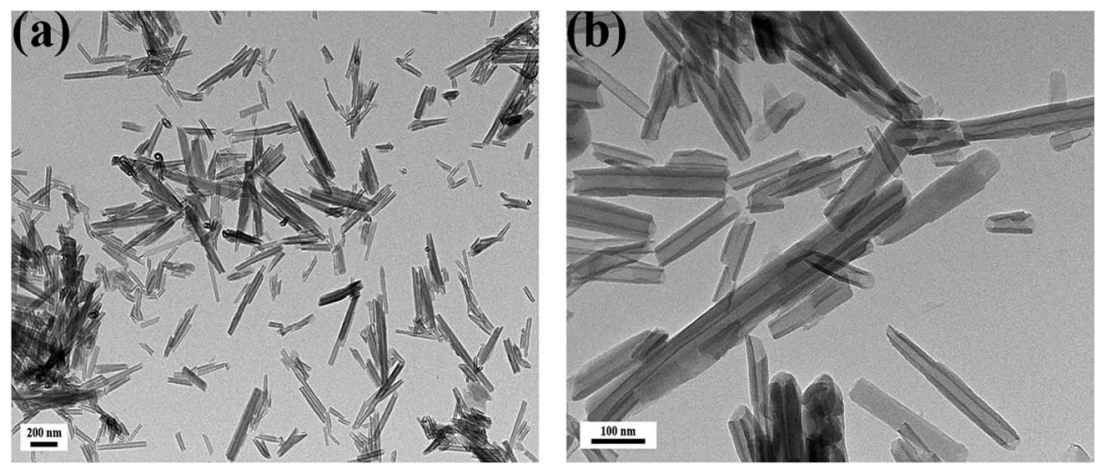

Fig. 1 TEM micrograph of halloysite nanotubes at different magnifications. Scale bars: $250 \mathrm{~nm}$ (a) and $100 \mathrm{~nm}$ (b). 

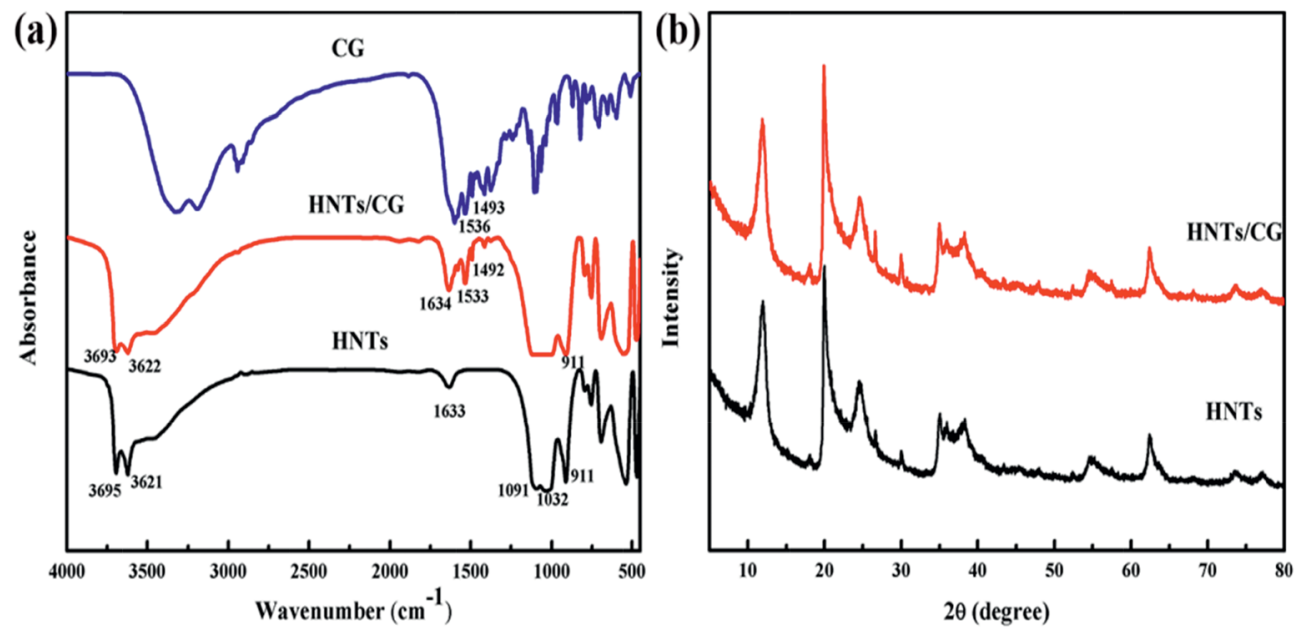

Fig. 2 FTIR spectra of HNTs, CG and HNTs/CG composite (a) and X-ray diffraction pattern of HNTs and HNTs/CG composite (b).
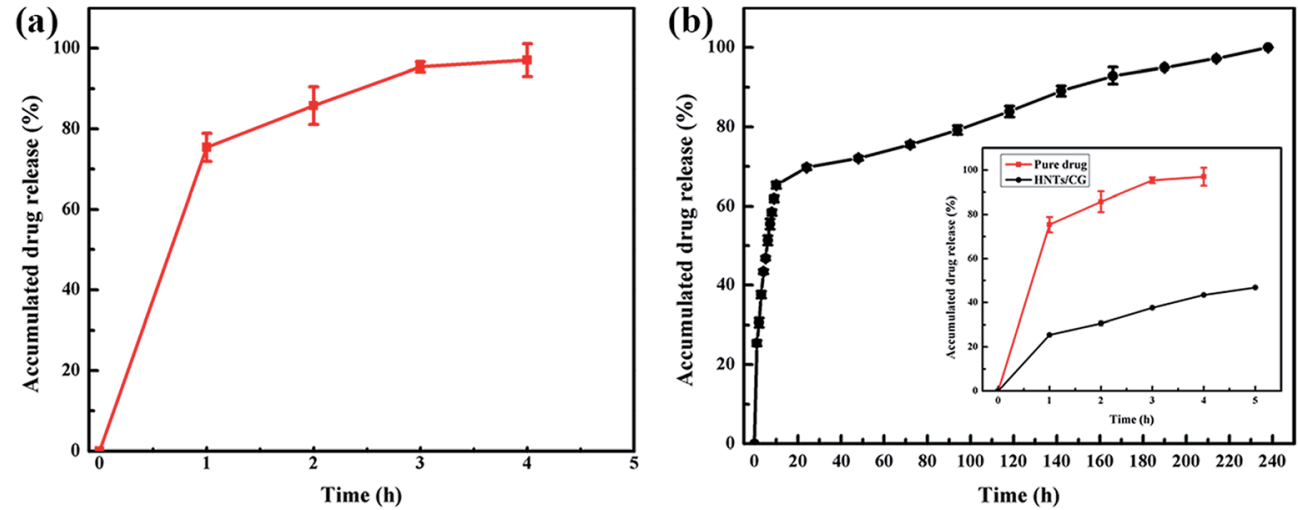

Fig. 3 In vitro release profiles of pure drug from dialysis membrane (a) and drug from HNTs/CG composite (b); the inset shows the release of pure drug from dialysis membrane and drug from HNTs/CG in the initial 5 hours.

method. The inhibition zone test results in Fig. 4 clearly show that neat HNTs did not display any inhibition zones for $S$. aureus, E. coli and $P$. aeruginosa. In contrast, the HNTs/CG
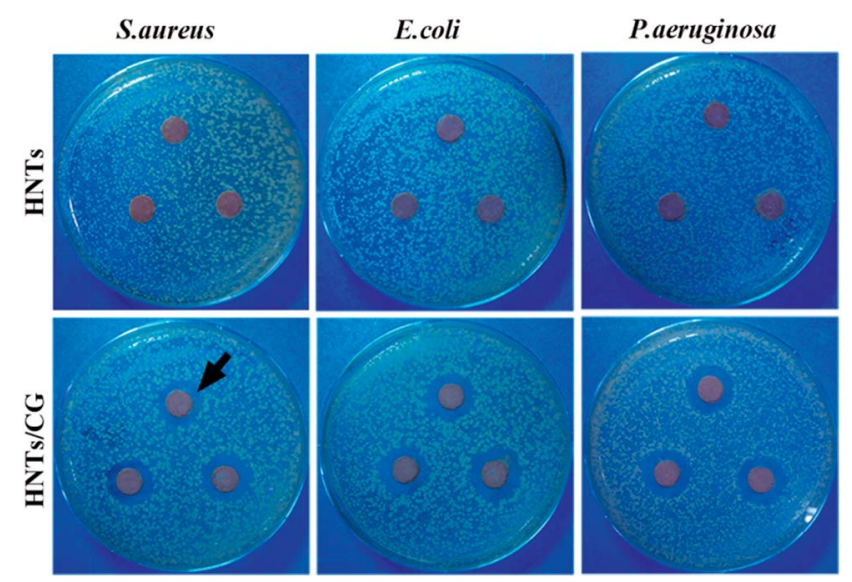

Fig. 4 Photographs of inhibition zone test of HNTs and HNTs/CG against $S$. aureus, E. coli and $P$. aeruginosa. composite showed very obvious inhibition zones around the specimen, and the diameter of each transparent zone was approximately $10 \mathrm{~mm}$. This phenomenon was attributed to the diffusion of CG on the plate, which inhibited the growth of $S$. aureus, E. coli and $P$. aeruginosa. The results suggested that CG was effectively loaded on HNTs, and endowed them with excellent antibacterial effect against Gram-positive and Gramnegative bacteria. In addition, the antibacterial activity of the HNTs/CG composite after one week release was also studied (Fig. S2 $\dagger$ ). After incubating the agar plates for $24 \mathrm{~h}$, the inhibition rings were still visible. This result indicated that the HNTs/ CG composite had long-term bacterial inhibition activity, which further demonstrated the controlled release effect of CG from HNTs.

\section{SEM observation of cotton fabric}

Then, we coated the HNTs/CG composite on cotton fabric to obtain a new textile that has long-lasting antibacterial properties to prevent contamination by bacteria. The surface morphology of raw cotton fabric and HNTs/CG-coated cotton fabric is shown in Fig. 5. As presented in the SEM images, it was 

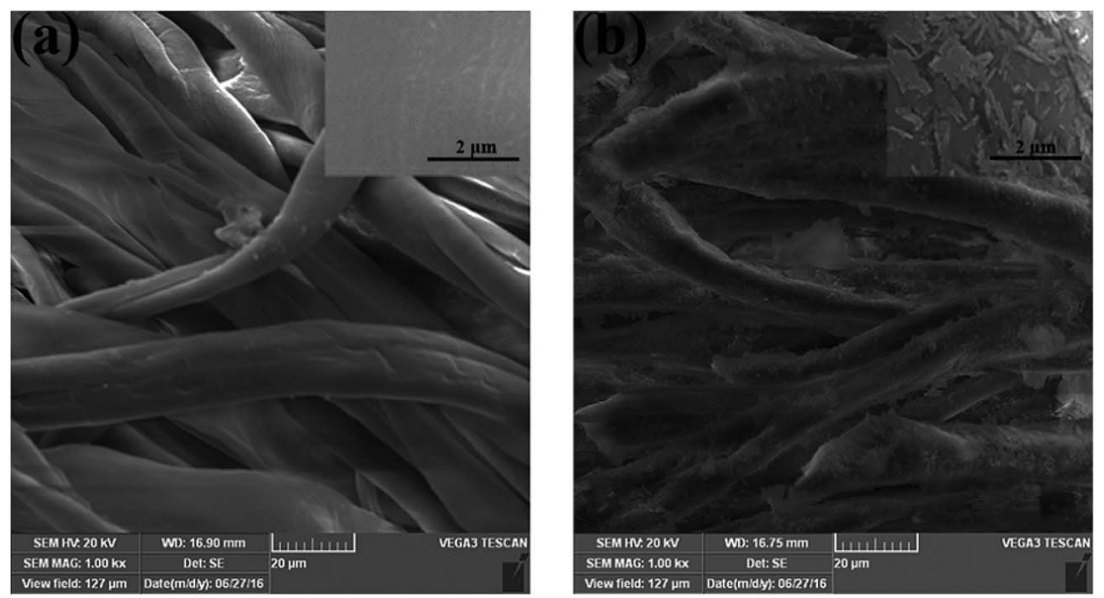

Fig. 5 SEM micrograph of raw cotton fabric (a) and HNTs/CG-coated cotton fabric (b). Scale bars in the main micrographs: $20 \mu$ m; scale bars in the insets: $2 \mu \mathrm{m}$.

found that the raw cotton had a smooth and uniform surface. After being finished with HNTs/CG, the surface of the fabric was rough. It was also observed at a high magnification that a spinal structure absorbed on the fabric surface. The higher magnification showed that the cotton fiber was covered with dispersed or aggregated nanotubes. Considering the fact that silanol groups ( $\mathrm{Si}-\mathrm{OH})$ are distributed on the external surface of HNTs, these could interact with the hydroxyl group $(-\mathrm{OH})$ of cotton fiber. The formation of hydrogen bonds between $\mathrm{Si}-\mathrm{OH}$ groups and - $\mathrm{OH}$ groups imparted the cotton textile with good durability against laundering. The durability was verified after 20 washing cycles. It could be clearly seen by SEM that a portion of HNTs/CG had been washed out, but some HNTs/CG were still tightly attached on the fiber surface (Fig. S3†). Several researchers have thought that HNTs could form a network structure through hydrogen bonding between the hydroxyls of the organics and oxygen atoms of $\mathrm{Si}-\mathrm{O}-\mathrm{Si}$ on the surfaces of HNTs, and the process was expected to facilitate the dispersion of HNTs in the matrix. In addition, the assembled inorganic network could partially take up the stress on the organic substance, resulting in significantly improved mechanical performance of the composites. ${ }^{39}$

\section{Thermal stability of HNTs/CG-coated cotton fabric}

The influence of HNTs/CG on the thermal stability of cotton fabric was investigated by thermogravimetric analysis (TGA). As shown in Fig. 6, the weight loss temperature, weight loss rate, and residual weight could be observed from TGA and derivative thermogravimetric (DTG) curves during the degradation process. ${ }^{40}$ At the first stage, the raw cotton fabric and HNTs/CGcoated cotton fabric showed a little weight loss when the temperature was below $250{ }^{\circ} \mathrm{C}$. Initial weight loss could be attributed to the dehydration of the cotton fabric. ${ }^{41}$ It was found that the major mass loss of both cotton fabrics took place in the temperature range of $250-400{ }^{\circ} \mathrm{C}$. The maximum pyrolysis rate occurred at the temperature of $357^{\circ} \mathrm{C}$ for raw cotton fabric and at $363{ }^{\circ} \mathrm{C}$ for the HNTs/CG-coated cotton. At this stage, it was due to the degradation of saccharide rings and the breakage of molecular chains. When the temperature was above $400{ }^{\circ} \mathrm{C}$, the degradation speed was much slower until $650{ }^{\circ} \mathrm{C}$; the residual weight of the raw cotton fabric and the HNTs/CG-coated cotton fabric was $9.7 \%$ and $13.9 \%$, respectively. These results indicated that HNTs could increase the charring products of the cotton fabric and consequently lead to enhanced flame retardancy of
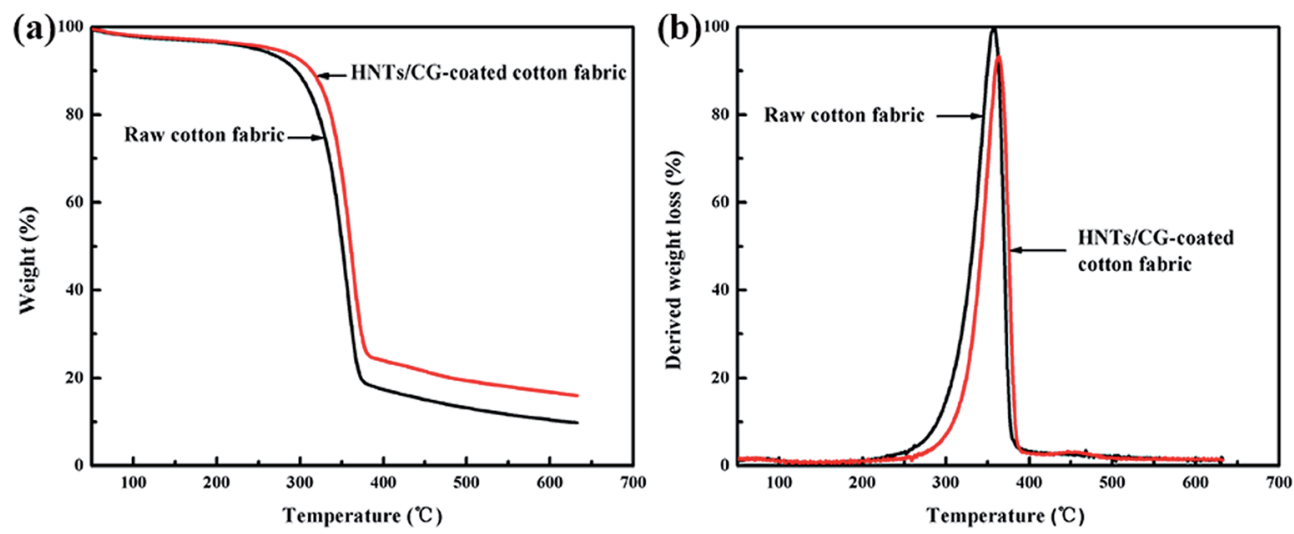

Fig. 6 TGA (a) and DTG curves (b) of raw cotton fabric and HNTs/CG-coated cotton. 
the cotton, and therefore the thermal stability of the cotton fabric had been improved.

The reasons why HNTs/CG could increase the thermal stability of polymers may be explained as follows. First, HNTs/ CG could be used as a flame retardant for polymers, exerting a barrier effect toward both mass and heat transport; therefore, adding HNTs/CG increased the thermal resistance of polymers. Secondly, the tubular structure could impede the diffusion of thermal decomposition products toward flame and oxygen by forming a spatial network, and thus delay the mass transport significantly. Moreover, the entry of degradation products into the lumen of HNTs would also hinder the mass transport and improve the thermal stability. ${ }^{\mathbf{4 2}}$

\section{Hydrophilicity of HNTs/CG-coated cotton fabric}

As we know, cotton is a kind of breathable fiber and able to absorb sweat into the clothing from the body and provide comfort to users. Here, we investigated the hydrophilicity of textiles and measured the water absorption rate. The capillary effects of raw cotton fabric and HNTs/CG-coated cotton fabric are shown in Fig. 7. It was found that the water absorption rate of cotton fabric finished with HNTs/CG was faster than the control sample, which manifested that HNTs/CG led to completely different wettability of the cotton fabric surface. The possible mechanism of the improvement of hydrophilicity could be explained as follows. Halloysite is a hydrophilic material with a $10^{\circ}$ water contact angle. ${ }^{42}$ When water was absorbed onto the hydrophilic surface of HNTs/CG-coated cotton textile, it was transferred to the fabric due to capillary penetration. The driving force for capillary penetration was the hydrophilicity of the fabric, which strongly relied on the surface energy and surface roughness. ${ }^{43}$ The hydrophilicity was better when the water wicking height was higher. ${ }^{\mathbf{4 4}}$ Hence, it could be concluded that the hydrophilicity of cotton fabric was improved through the HNTs/CG finishing.

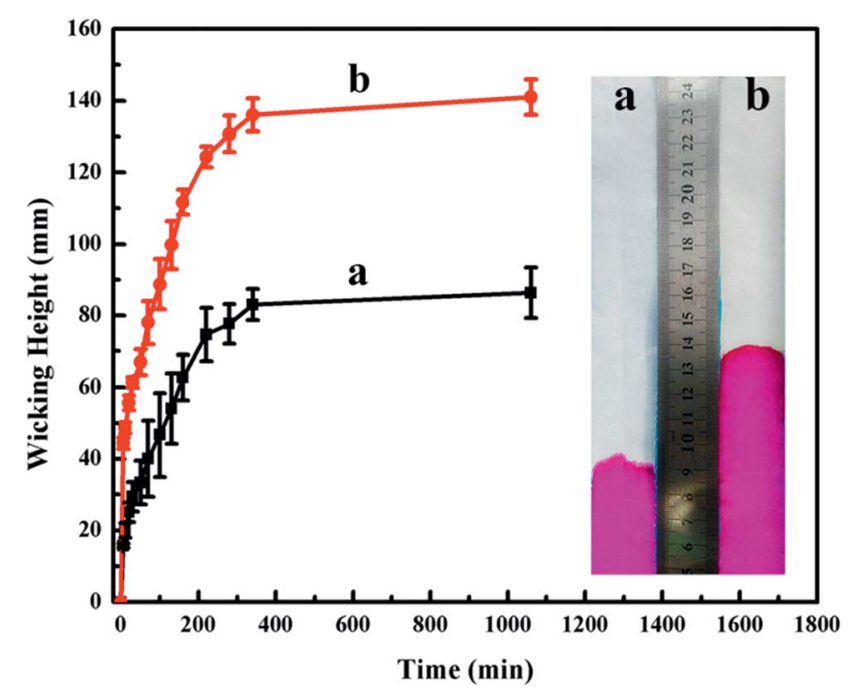

Fig. 7 Capillary effect of raw cotton fabric (a) and HNTs/CG-coated cotton fabric (b).

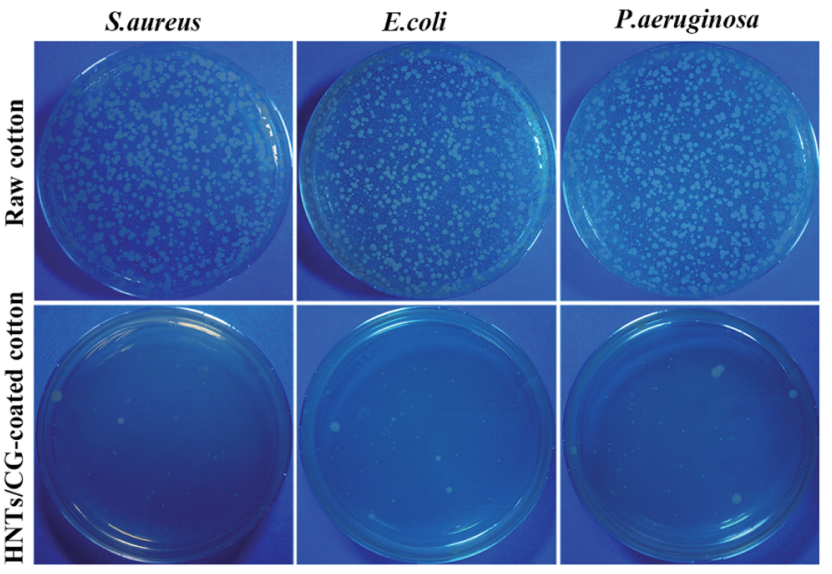

Fig. 8 Antibacterial activities of HNTs/CG-coated cotton fabric against $S$. aureus, $E$. coli and $P$. aeruginosa. The raw cotton fabric served as a control.

\section{Antibacterial test of HNTs/CG-coated cotton fabric}

Antibacterial rate was used to quantitatively evaluate the antibacterial activity of HNTs/CG-coated cotton fabric by the viable cell counting technique. ${ }^{45}$ The antibacterial effect of HNTs/CG-coated cotton fabric against $S$. aureus, $E$. coli and $P$. aeruginosa is shown in Fig. 8. After the 24 hour co-culture of bacteria and the raw cotton fabric, the agar plates were covered with bacteria colonies. However, the count for viable colonies of bacteria was significantly decreased at the same time when co-cultured with HNTs/CG-coated cotton fabric. The results showed that the antibacterial rates against $S$. aureus, E. coli and $P$. aeruginosa were about $99.10 \%, 98.29 \%$ and $98.33 \%$, respectively. This implied that the resultant cotton fabric finished with HNTs/CG composite possesses outstanding antibacterial activity against both Gram-positive and Gramnegative bacteria.

Actually, the durability of antibacterial effect is a crucial issue in the production of antibacterial textiles. In view of the importance of this issue, the antibacterial effect of HNTs/CGcoated cotton fabric after 20 washing cycles was measured. As seen in Table 1 , the antibacterial activity of HNTs/CG-coated cotton fabric against all tested microorganisms still remained at over $90 \%$ bacterial reduction, indicating that the HNTs/CGcoated cotton fabric showed excellent antibacterial activity and washing durability against repeated laundering.

The antibacterial activity was further confirmed by cell viability assays (Fig. $\mathrm{S} 4 \dagger$ ). Almost all the E. coli exposed to raw cotton were stained a green color, while the cells treated with

Table 1 Antibacterial activities of HNTs/CG-coated cotton before and after washing

\begin{tabular}{llll}
\hline & \multicolumn{2}{l}{ Antibacterial rate $(\%)$} \\
\cline { 2 - 4 } Sample & S. aureus & E. coli & P. aeruginosa \\
\hline Initial & 99.02 & 98.29 & 98.32 \\
Washed 20 times & 94.62 & 90.06 & 91.61
\end{tabular}


the HNTs/CG-coated cotton were stained in red. This indicated that the HNTs/CG-coated cotton has prominent antibacterial activity, which was consistent with the results from the antibacterial rate test before. The antibacterial mechanism of the HNTs/CG-coated cotton fabric against E. coli was preliminarily studied by SEM observation of cells.

From the SEM images (Fig. S5 $\dagger$ ), it could be found that $E$. coli co-cultured with raw cotton fabric for $24 \mathrm{~h}$ was still intact and rod-shaped, whereas $E$. coli exposed to HNTs/CG-coated cotton lost their cellular integrity. The antibacterial mechanism of HNTs/CG may be interpreted as follows: the negatively charged cell walls could interact electrostatistically with the positively charged drug and disturb the membrane, therefore leading to the efflux of intracellular contents and the demise of bacteria. ${ }^{46}$

\section{Skin irritation test}

Toxicity is an essential factor in the practical application of the antibacterial textile. Skin stimulation testing was conducted here to evaluate the sensitization due to antibacterial textile. According to the technical standard for disinfection, the antibacterial HNTs/CG composite was applied to the rabbits' backs and covered with 4 layers of gauze; neither erythema nor edema was observed after 1, 24, 48 and $72 \mathrm{~h}$ (Fig. 9), and the scores of stimulus response were 0 in every tested rabbit (Table S1 $\dagger$ ). The results showed that the HNTs/CG composite caused no irritation to rabbits' skins.

\section{Cytotoxicity test}

The cytotoxicity of raw cotton fabric and HNTs/CG-coated cotton fabric was evaluated through MTT test. The extraction medium was cultured with mouse fibroblast cells (L929) for 24, 48 and 72 h. As shown in Fig. 10, the raw cotton fabric showed no significant difference from the control cells at $24 \mathrm{~h}$ as well as at 48 and $72 \mathrm{~h}$. It could be seen that the viability of cells treated with HNTs/CG-coated cotton fabric was $95 \%, 79 \%$ and $85 \%$ of the negative control at 24,48 and $72 \mathrm{~h}$, respectively. The viability of cells treated with raw cotton fabric was slightly higher than that of HNTs/CG-coated cotton. As reported before, the average relative cell viability was over $70 \%$, indicating the low toxicity of HNTs/CG-coated cotton material. ${ }^{47}$ These results indicated that the HNTs/CG-coated cotton fabric had low toxicity to L929 cells. In our previous work, we investigated the toxicity of HNTs on A549 cells by CCK-8 test and TUNEL (terminal deoxynucleotidyl

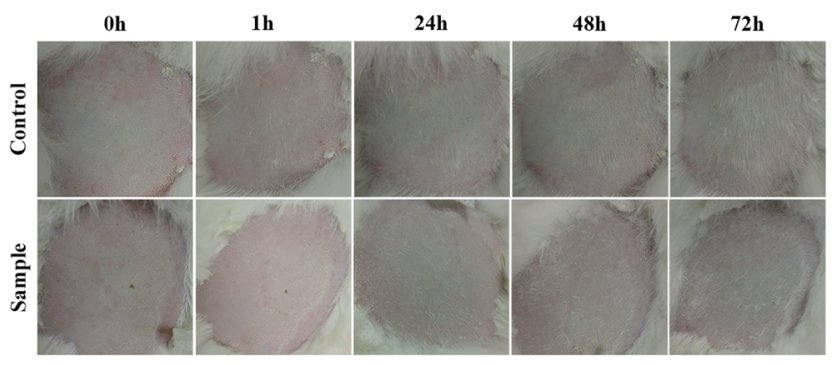

Fig. 9 Photographs of animals' skin stimulation at $0,1,24,48$ and $72 \mathrm{~h}$ One side of the rabbits was treated with HNTs/CG composite, and the other side was regarded as a control.

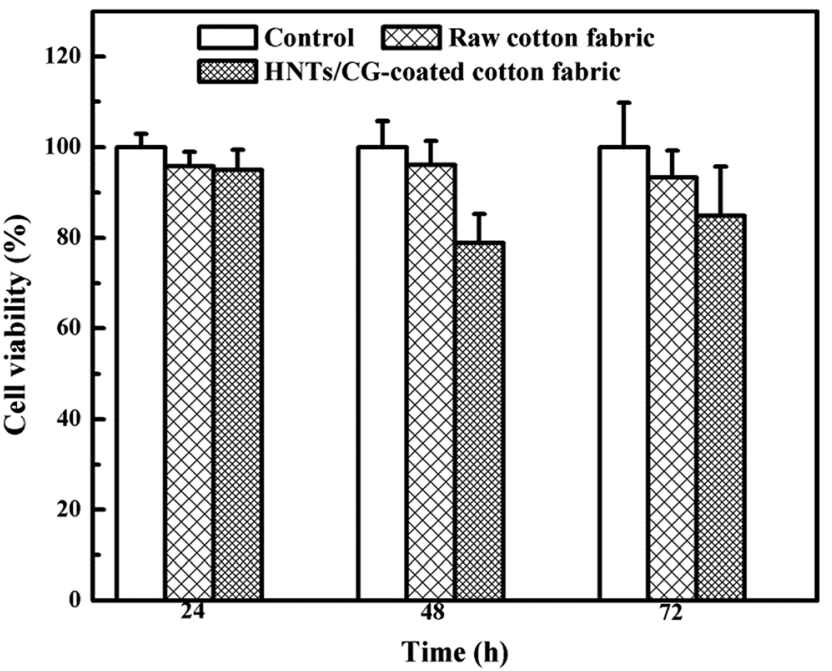

Fig. 10 In vitro cytotoxicity tests of L929 cells cultured in extracts of raw cotton fabric and HNTs/CG-coated cotton fabric for 24, 48 and $72 \mathrm{~h}$. The data are represented as mean \pm standard deviation (SD).

transferase dUTP nick end labeling) assay, showing a high biocompatibility at a lower concentration. ${ }^{48}$ Lvov \& Abdullayev ${ }^{49}$ believed that the toxicity may relate to the length of HNTs. From their point of view, the shorter HNTs could be internalized by macrophages, and they thought that the direct toxicity of halloysite to biocells would not be an issue in applications. Nevertheless, further research is required to investigate the possible long-term toxic effects of halloysite nanotubes in vivo and in vitro.

\section{Conclusions}

In general, the CG-loaded halloysite nanotubes were successfully prepared. The CG could be released from HNTs/CG composite in a sustained manner. The initial HNTs/CG composite showed high bacterial inhibition activity against $S$. aureus, E. coli, and $P$. aeruginosa. After one week release, the HNTs/CG still exhibited significant antibacterial activity. HNTs/ CG-coated cotton was also successfully obtained with HNTs/CG composite as an antibacterial coating. The HNTs/CG-coated cotton fabric exhibited improved hydrophilicity, thermal stability, and excellent antibacterial effect compared with the raw cotton fabric. The HNTs/CG-coated cotton possessed good biocompatibility and showed no stimulation to the skin of rabbits and nontoxicity to L929 cells. Therefore, the HNTs/CGcoated cotton fabric is a promising antibacterial material and will have broad application in the textile field.

\section{Acknowledgements}

This work was supported by National Tertiang Student Renovation Experiment Project Fund of the Chinese Ministry of Education (No. 2042014gf036). 


\section{References}

1 Y. Liu, K. Ma, R. Li, X. Ren and T. S. Huang, Cellulose, 2013, 20, 3123-3130.

2 Y. Chen, Y. Zhang, H. Zhang, J. Liu and C. Song, Chem. Eng. J., 2013, 228, 12-20.

3 L. Windler, M. Height and B. Nowack, Environ. Int., 2013, 53, 62-73.

4 M. H. EI-Rafie, H. B. Ahmed and M. K. Zahran, Carbohydr. Polym., 2014, 107, 174-181.

5 M. K. Zahran, H. B. Ahmed and M. H. EI-Rafie, Carbohydr. Polym., 2014, 108, 145-152.

6 A. M. Gallardo-Moreno, M. A. Pacha-Olivenza, M. C. Fernández-Calderón, C. Pérez-Giraldo, J. M. Bruque and M. L. González-Martín, Biomaterials, 2010, 31, 5159-5168.

7 W. A. Daoud, J. H. Xin and Y. H. Zhang, Surf. Sci., 2005, 599, 69-75.

8 P. Dhandapani, A. S. Siddarth, S. Kamalasekaran, S. Maruthamuthu and G. Rajagopal, Carbohydr. Polym., 2014, 103, 448-455.

9 N. Vigneshwaran, S. Kumar, A. A. Kathe, P. V. Varadarajan and V. Prasad, Nanotechnology, 2006, 17, 5087-5095.

10 S. Hendessi, E. B. Sevinis, S. Unal and F. C. Cebeci, Prog. Org. Coat., 2016, 101, 253-261.

11 G. Cavallaro, D. I. Donato, G. Lazzara and S. Milioto, J. Phys. Chem. C, 2011, 115, 20491-20498.

12 L. Yu, H. X. Wang, Y. T. Zhang, B. Zhang and J. D. Liu, Environ. Sci.: Nano, 2016, 3, 28-44.

13 X. X. Ding, H. X. Wang, W. H. Chen, J. D. Liu and Y. T. Zhang, RSC Adv., 2014, 4, 41993-41996.

14 F. R. Ahmed, M. H. Shoaib, M. Azhar, S. H. Um, R. I. Yousuf, S. Hashmi and A. Dar, Colloids Surf., B, 2015, 135, 50-55.

15 V. Vergaro, E. Abdullayev, Y. M. Lvov, A. Zeitoun, R. Cingolani, R. Rinaldi and S. Leporatti, Biomacromolecules, 2010, 11, 820826.

16 R. Shemesh, M. krepker, M. Natan, Y. Danin-Poleg, E. Banin, Y. Kashi, N. Nitzan, A. Vaxman and E. Segal, RSC Adv., 2015, 5, 87108-87117.

17 M. Liu, Y. Zhang, C. Wu, S. Xiong and C. Zhou, Int. J. Biol. Macromol., 2012, 51, 566-575.

18 A. D. Hughes and M. R. King, Langmuir, 2010, 26, 1215512164.

19 J. Xue, Y. Niu, M. Gong, R. Shi, D. Chen, L. Zhang and Y. Lvov, ACS Nano, 2015, 9, 1600-1612.

20 Y. J. Suh, D. S. Kil, K. S. Chung and D. Mongayt, J. Nanosci. Nanotechnol., 2011, 11, 661-665.

21 Y. Y. Zhang, Q. B. Xu, F. Y. Fu and X. D. Liu, Cellulose, 2016, 23, 2791-2808.

22 W. Ye, M. F. Leung, J. Xin, T. L. Kwong, D. K. L. Lee and P. Li, Polymer, 2005, 46, 10538-10543.

23 N. Meng, N. L. Zhou, S. Q. Zhang and J. Shen, Int. J. Pharm., 2009, 382, 45-49.

24 Y. Gao and R. Cranston, Text. Res. J., 2008, 78, 60-72.
25 N. S. Fraser, A. K. Johnson, R. R. Wilborn, G. A. Dujovne and L. Nuehring, Theriogenology, 2017, 88, 61-66.

26 B. H. Kapadia, R. K. Elmallah and M. A. Mont, J. Arthroplasty, 2016, 31, 2856-2861.

27 H. Cai, F. Bao, J. Gao, T. Chen, S. Wang and R. Ma, Environ. Technol., 2015, 36(10), 1273-1280.

28 E. Abdullayev, R. Price, D. Shchukin and Y. Lvov, ACS Appl. Mater. Interfaces, 2009, 1, 1437-1443.

29 D. Fix, D. V. Andreeva, Y. M. Lvov, D. G. Shchukin and H. MÖhwald, Adv. Funct. Mater., 2009, 19, 1720-1727.

30 Y. Lvov, A. Aerov and R. Fakhrullin, Adv. Colloid Interface Sci., 2014, 207, 189-198.

31 M. R. Dzamukova, E. A. Naumenko, Y. M. Lvov and R. F. Fakhrullin, Sci. Rep., 2015, 5, 1-11.

32 S. R. Levis and P. B. Deasy, Int. J. Pharm., 2003, 253, 145-157. 33 Y. M. Lvov, D. G. Shchukin, H. Mhwald and R. Price, ACS Nano, 2008, 2, 814-820.

34 Y. Zhang, X. He, J. Ouyang and H. Yang, Sci. Rep., 2013, 3, 16.

35 R. Li, Q. He, Z. Hu, S. Zhang, L. Zhang and X. Chang, Anal. Chim. Acta, 2012, 713, 136-144.

36 R. Qi, R. Guo, F. Zheng, H. Liu, J. Yu and X. Shi, Colloids Surf., B, 2013, 110, 148-155.

37 C. Viseras, P. Cerazo, R. Sanchez, I. Salcedo and C. Aguzzi, Appl. Clay Sci., 2010, 48, 291-295.

38 E. M. G. Raso, M. E. Cortes, K. I. Teixeira, M. B. Franco, N. D. S. Mohallem and R. D. Sinisterra, J. Inclusion Phenom. Macrocyclic Chem., 2010, 67, 159-168.

39 M. Du, B. Gao and D. Jia, Polym. Int., 2010, 59, 574-582.

40 P. Zhu, S. Sui, B. Wang, K. Sun and G. Sun, J. Anal. Appl. Pyrolysis, 2004, 71, 645-655.

41 X. Feng, K. Zheng, C. Wang, F. Chu and Y. Chen, Fibers Polym., 2016, 17(13), 371-379.

42 M. Liu, Z. Jia, D. Jia and C. Zhou, Prog. Polym. Sci., 2014, 39, 1498-1525.

43 S. Chen, L. Yuan, Q. Li, J. Li, X. Zhu, Y. Jiang, Q. Sha, X. Yang, J. H. Xin, J. Wang, F. J. Stader and P. Huang, Small, 2016, 12, 3516-3521.

44 S. Chen, S. Chen, S. Jiang, M. Xiong, J. Luo, J. Tang and Z. Ge, ACS Appl. Mater. Interfaces, 2011, 3, 1154-1162.

45 I. Perelshtein, G. Applerot, N. Perkas, E. Wehrschetz-Sigl, A. Hasmann, G. M. Guebitz and A. Gedanken, ACS Appl. Mater. Interfaces, 2009, 1, 361-366.

46 M. Yu, Z. Wang, M. Lv, R. Hao, R. Zhao, L. Qi, S. Liu, C. Yu, B. Zhang, C. Fan and J. Li, ACS Appl. Mater. Interfaces, 2016, 8, 19866-19871.

47 W. Kangwansupamonkon, V. Lauruengtana, S. Surassmo and U. Ruktanonchai, J. Nanomed. Nanotechnol., 2009, 5, 240-249.

48 H. Y. Liu, L. Du, Y. T. Zhao and W. Q. Tian, J. Nanomater., 2015, 2, 1-9.

49 Y. Lvov and E. Abdullayev, Prog. Polym. Sci., 2013, 38, 16901719. 